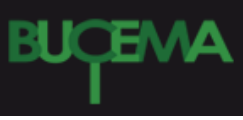

Bulletin du centre d'études médiévales d'Auxerre | BUCEMA

Hors-série $n^{\circ} 3 \mid 2010$

Présentation et mise en valeur des sites archéologiques religieux en milieu urbain

\title{
Aoste (Italie), Le complexe St. Ours - St. Laurent et le groupe épiscopal.
}

Renato Perinetti et Mauro Cortelazzo

\section{(2) OpenEdition}

Journals

Édition électronique

URL : https://journals.openedition.org/cem/11387

DOI : $10.4000 /$ cem. 11387

ISSN : 1954-3093

Éditeur

Centre d'études médiévales Saint-Germain d'Auxerre

Référence électronique

Renato Perinetti et Mauro Cortelazzo, « Aoste (Italie), Le complexe St. Ours - St. Laurent et le groupe épiscopal. », Bulletin du centre d'études médiévales d'Auxerre | BUCEMA [En ligne], Hors-série n 3 | 2010, mis en ligne le 15 mars 2010, consulté le 02 mars 2023. URL : http://journals.openedition.org/cem/ 11387 ; DOl : https://doi.org/10.4000/cem. 11387

Ce document a été généré automatiquement le 2 mars 2023.

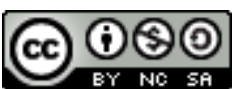

Creative Commons - Attribution - Pas d'Utilisation Commerciale - Partage dans les Mêmes Conditions 4.0 International - CC BY-NC-SA 4.0

https://creativecommons.org/licenses/by-nc-sa/4.0/ 


\title{
Aoste (Italie), Le complexe St. Ours - St. Laurent et le groupe épiscopal.
}

\author{
Renato Perinetti et Mauro Cortelazzo
}

\section{Premesse.}

1 La sistemazione e l'apertura al pubblico dei siti archeologici realizzati dalla Soprintendenza regionale per i Beni e le Attività Culturali della valle d'Aosta, sono il risultato di collaborazioni, riflessioni e dibattiti svoltisi in questi ultimi decenni nei vari cantieri in cui si è operato e della particolare situazione amministrativa della nostra Regione ${ }^{1}$.

2 Le scelte e le soluzioni adottate tengono conto, oltre che delle specificità proprie di ogni scavo, anche delle particolari condizioni ambientali e climatiche del nostro territorio, che certamente non sono favorevoli alla conservazione delle vestigia all'aperto.

3 La musealizzazione dei siti archeologici, operazione quanto mai complessa e stimolante, in quanto coinvolge problematiche diverse, non è altro che la scelta critica del "messaggio" che si intende rivolgere al fruitore. Il "messaggio", come tale, tiene conto delle esigenze imposte dalla ricerca, dalla conservazione e dal restauro che insieme rendono possibile il testo didattico.

4 La leggibilità e la comprensione, da parte del pubblico, di un sito archeologico pluristratificato, può essere realizzata con la "semplificazione" del messaggio "privilegiando" alcune delle fasi costruttive del monumento. Naturalmente bisognerà tenere conto anche della valenza territoriale dei ritrovamenti e dello stato di conservazione dei reperti. Non è certamente nostra intenzione proporre la distruzione fisica dei resti basata su presupposti preferenziali, estetici o tecnici, bensì l'attenuazione o la sottolineatura, con operazioni controllate, di tutto quanto intendiamo lasciare in ombra o mettere in evidenza. E' inoltre opportuno tener conto che qualsiasi scavo archeologico è, per definizione, un intervento distruttivo, anche se controllato e documentato in tutte le sue fasi e che il risultato finale è mediato e 
condizionato dalle scelte che effettua l'archeologo, scelte che devono essere "guidate" da appropriate problematiche di scavo che prevedano la possibilità di conservare in situ i ritrovamenti, in funzione della loro "trasmissione al futuro". In alcuni casi è opportuno, anche a scapito della completezza dello scavo, evitare lo smontaggio di pavimenti e strutture al fine di agevolarne la fruizione ${ }^{2}$. Per questi motivi la scelta di rendere fruibili i siti archeologici, deve essere compiuta prima di iniziare l'intervento di ricerca. E' inoltre necessario disporre, all'interno della struttura preposta all'intervento, di specialisti in grado di bloccare sul nascere i processi degenerativi propri di tutte le strutture che emergono dal terreno e che, proprio a partire dal momento del rinvenimento, rischiano i danni maggiori. Spesso interventi non tempestivi pregiudicano la consistenza fisica di un manufatto, innescando un irreversibile processo distruttivo limitando poi di fatto qualsiasi possibilità didattica futura: è nota, la fragilità delle malte in genere, degli intonaci, dei pavimenti, ecc. La presenza del restauratore in cantiere rende possibile la realizzazione di scavi in "sicurezza" e crea le premesse per il restauro definitivo.

5 La comprensione del messaggio e la leggibilità di un "testo" archeologico possono essere affidate, almeno in parte, ad operazioni "complementari" come ad esempio il risarcimento delle lacune, mediante l'impiego di materiali appropriati e riconoscibili, e la predisposizione di documenti illustrativi (piante, sezioni, ricostruzioni tridimensionali, modellini, ecc.).

6 Particolare attenzione va posta nell'individuazione dei percorsi di visita sotterranei che devono costantemente evidenziare il collegamento scavo-monumento in elevato e le varie fasi costruttive.

7 Gli interventi, così come abbiamo sommariamente accennato, presuppongono l'esistenza di alcune condizioni operative: la scelta, prima dell'avvio degli scavi, di rendere accessibile al pubblico il sito prescelto, l'accettazione, da parte delle Amministrazioni interessate, di programmi e impegni a lungo termine, la preparazione di operatori specializzati sia dal punto di vista tecnico che scientifico, ed infine la garanzia della continuità e adeguatezza dei finanziamenti. Si sottolinea inoltre la necessità di individuare, almeno all'inizio dei lavori, i progettisti delle opere di sistemazione, al fine di coinvolgerli nelle problematiche della ricerca e della conservazione, coinvolgimento che, se effettuato in corso d'opera, può costituire valido momento di confronto.

8 Nel caso di interventi all'interno di monumenti, come ad esempio le chiese, bisognerà anche prevedere il finanziamento del restauro dell'edificio che sarà effettuato sulla base dei ritrovamenti archeologici. Impegni tecnici ed economici di così vasta portata implicano un ritorno in termini costi-benefici ${ }^{3}$, in cui i benefici non possono essere che di tipo culturale e quindi individuabili nel recupero della memoria storica di una comunità nel senso di conoscenza del proprio passato. In ultima analisi si tratta di instaurare un rapporto di dialogo, tra i ricercatori e gli utenti destinatari dei nostri lavori ${ }^{4}$. Occorre cioè finalizzare la ricerca, lo studio, il restauro e infine la fruibilità del contesto indagato allo scopo di dare comunicazione dei risultati, poiché la crescita di un settore di un settore come quello dei Beni Culturali avviene solo attraverso un costante processo di riappropriazione da parte di chi quegli spazi li vive, prima e dopo gli interventi, ogni giorno.

9 Sulla base di queste premesse sono stati programmati interventi su due siti di fondamentale importanza per la nostra Regione, la Cattedrale e il complesso di S. Orso, 
il primo nel centro politico-amministrativo dell'antica città romana e il secondo in una delle necropoli orientali fuori Porta Praetoria. La ricerca archeologica aveva come obiettivo lo studio della nascita dei primi edifici sacri cristiani e il restauro dei due complessi monumentali ${ }^{5}$.

Occorre premettere che all'epoca (anno 1972) erano note esclusivamente due epigrafi che citavano i vescovi Gratus e Gallus e un piccolo edificio religioso rinvenuto fuori Porta Decumana nel 1939. La lista epicopale contava, per il primo millennio, solamente 9 vescovi (Eustasius, morto dopo il 451, Grato, morto nella seconda metà del v secolo, Giocondo, morto verso il 510, un vescovo anonimo ricordato in una lettera di Teodorico datata tra il 511 e il 518, Gallus, morto nel 529, Plotianus, ricordato nella Vita Beati Ursi e vissuto tra il VI e l'viII secolo, Ratbornus che prese parte al sinodo di Ravenna dell'877, Liutfredus che firma gli atti del sinodo milanese del 969 e infine Anselmo, morto nel $1026^{6}$.

Gli scavi hanno invece permesso di verificare l'esistenza della prima cattedrale già alla fine del IV secolo e la costruzione delle chiese funerarie fuori le mura nella prima metà del v secolo (fig. 1).

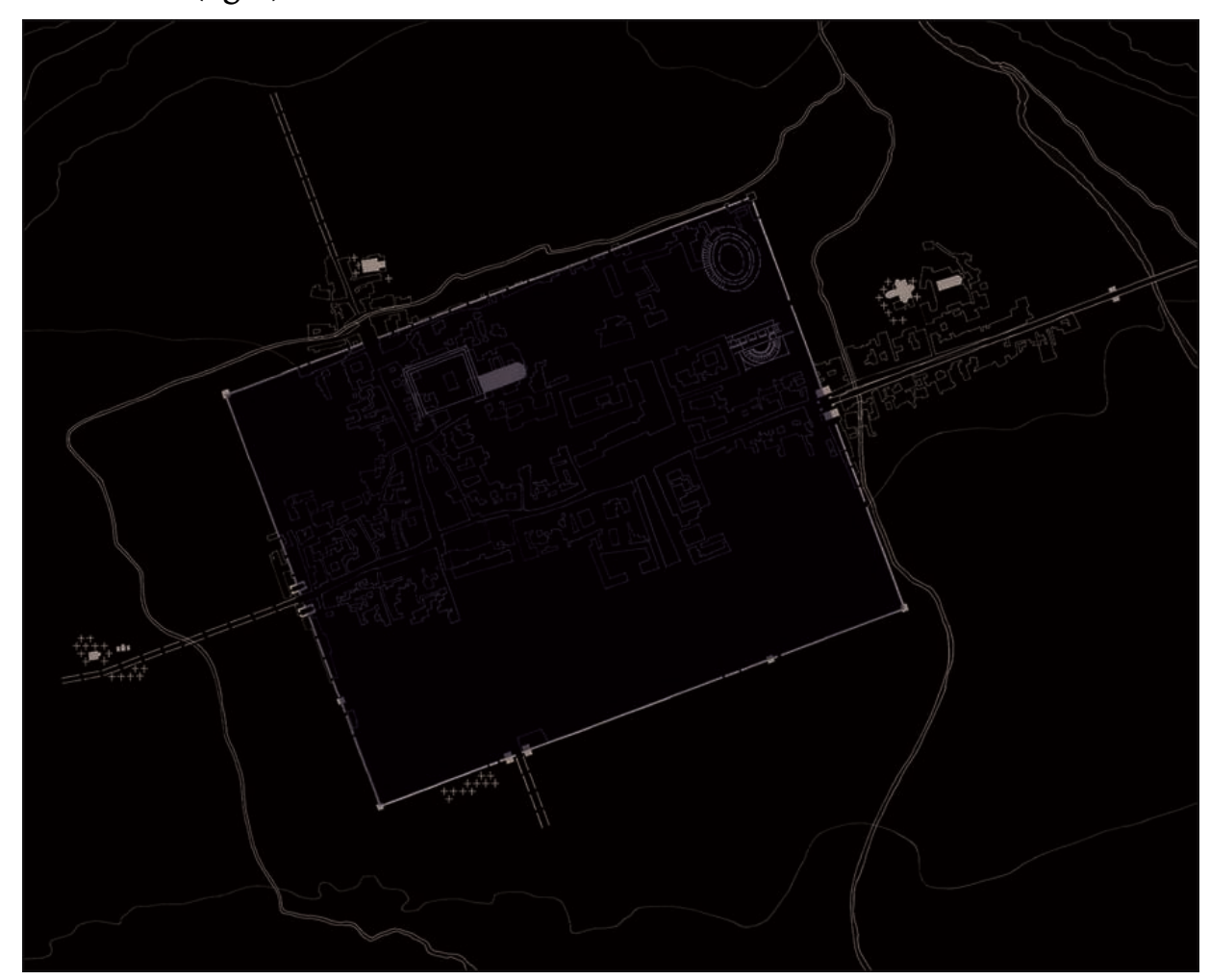

Fig. 1 - Planimetria generale.

\section{Complesso di S. Orso}

A partire dal 1972, a seguito di una Tavola Rotonda che aveva riunito illustri studiosi italiani ed europei per dibattere le metodiche di ricerca e restauro del sito, si è proceduto alla realizzazione dello scavo archeologico delle chiese di S. Lorenzo e di S. Orso, di parte del chiostro, e di alcune aree settentrionali limitrofe, in preparazione degli interventi di valorizzazione del complesso monumentale. Tutti gli scavi sono stati eseguiti senza interrompere le funzioni liturgiche all'interno degli edifici sacri. I 
risultati hanno permesso di riportare alla luce i resti di due chiese a pianta cruciforme che rimandano agli esempi della basilica Apostolorum e S. Simpliciano fatti costruire dal santo vescovo Ambrogio a Milano, nell'ultimo quarto del IV secolo. Il complesso sarà completamente ricostruito nel IX secolo e nuovamente all'inizio dell'xi (figg. 2, 3 e 4). La chiesa di $\mathrm{S}$. Lorenzo avrà ancora un'ulteriore importante fase ricostruttiva verso la metà del XVII secolo.

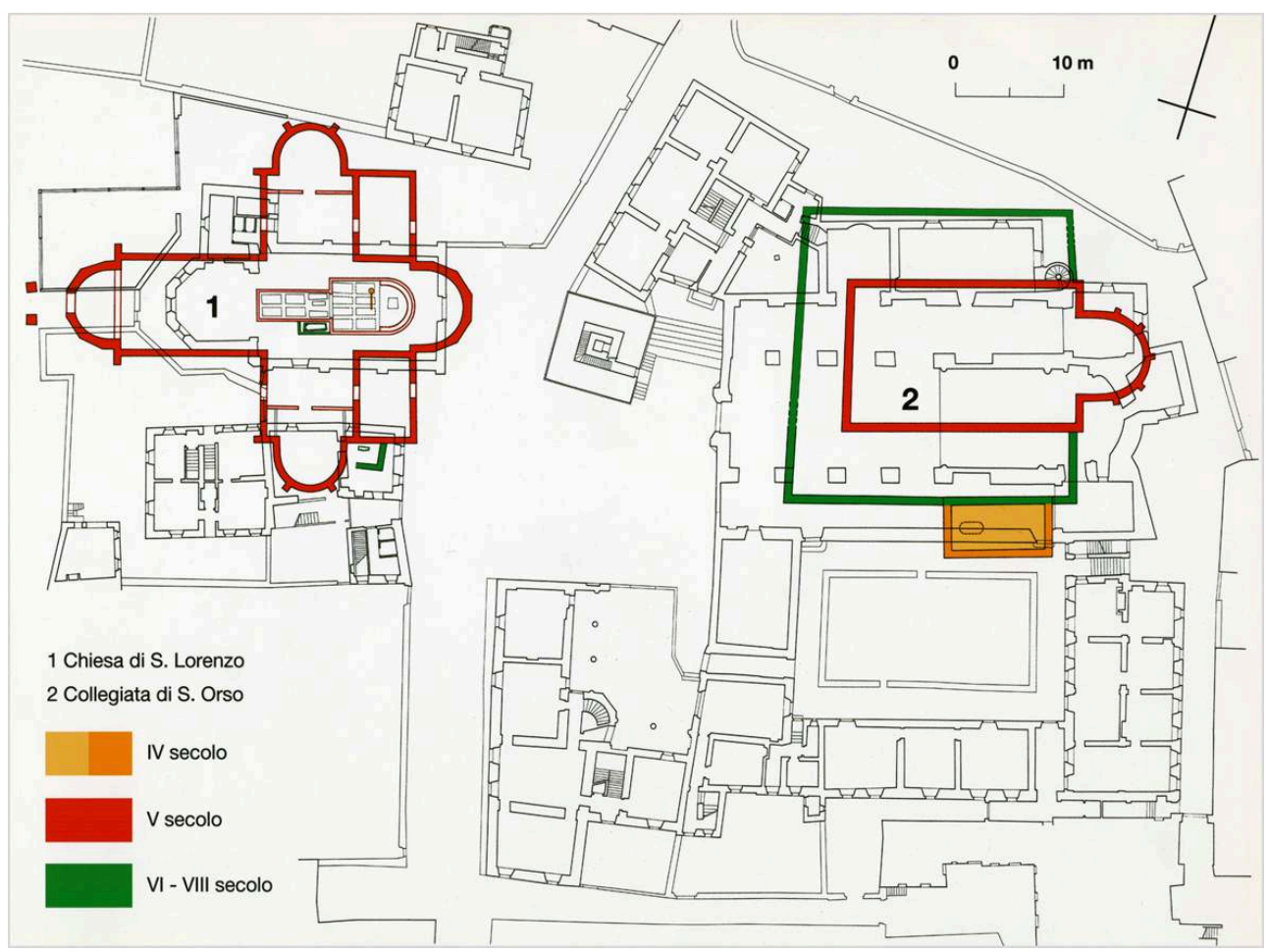

Fig. 2 - Complesso di S. Orso: Chiese paleocristiane. 


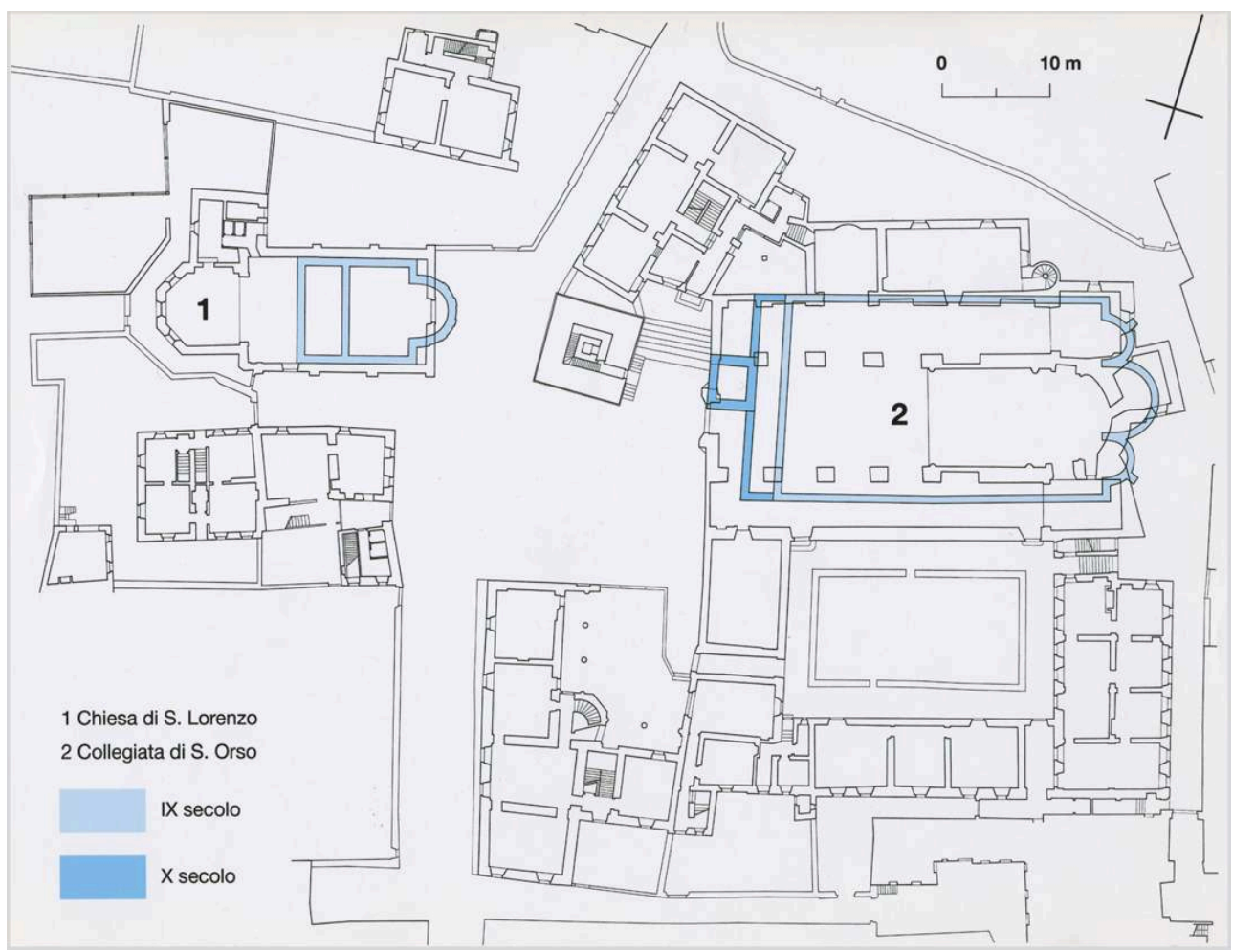

Fig. 3 - Cmplesso di S. Orso: Chiese altomedievale.

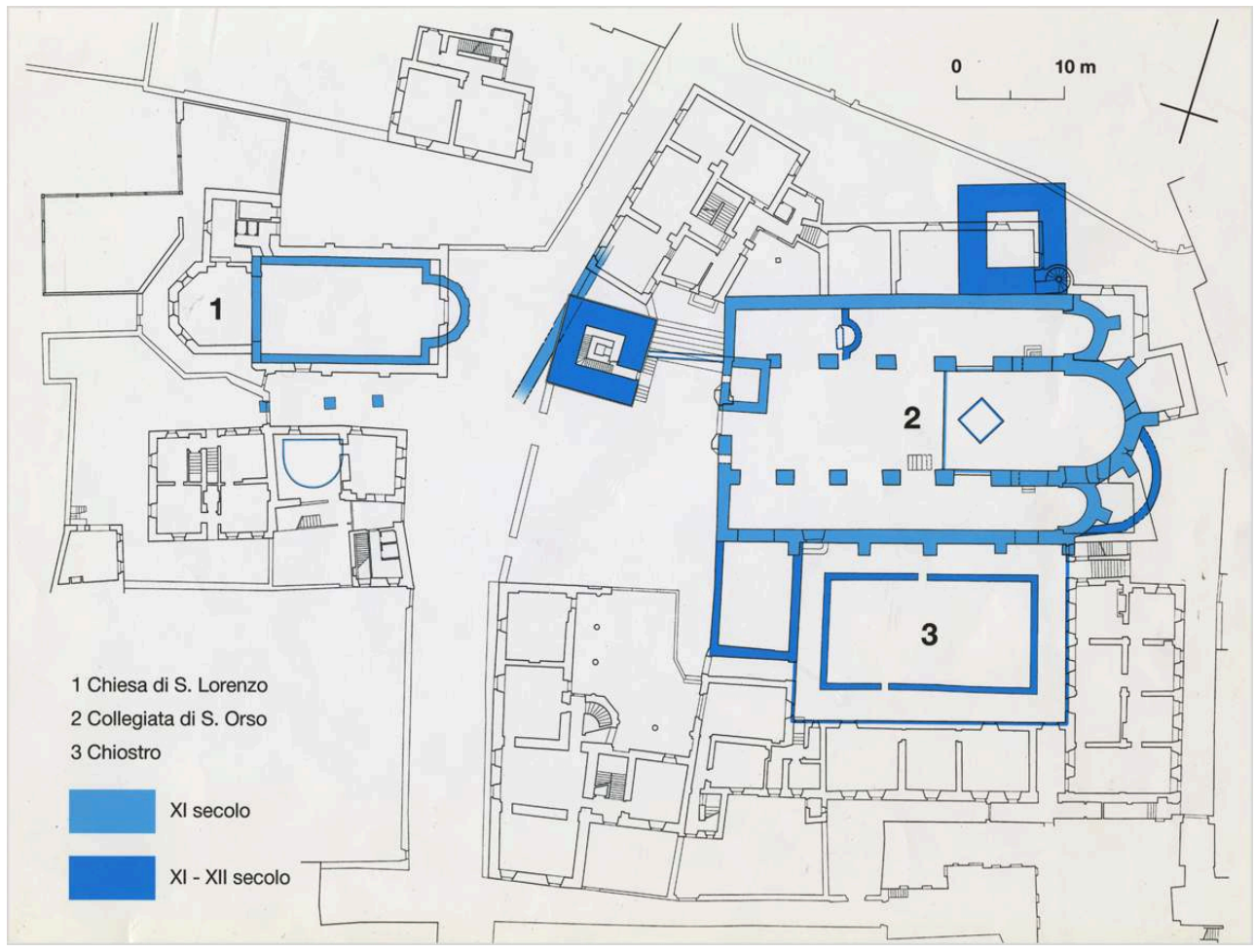

Fig. 4 - Complesso di S. Orso: Chiese xı secolo.

Le ricerche hanno impegnato la Soprintendenza regionale per ben 4 decenni ed è tutt'ora in corso lo scavo dell'area antistante la chiesa di S. Orso. 
14 Attualmente è in fase di preparazione un disegno di legge per finanziare e programmare il restauro completo del complesso e l'avvio di ulteriori scavi archeologici nel settore più a nord del complesso.

In questi ultimi anni si è invece proceduto alla sistemazione della visita agli affreschi del sottotetto della chiesa di S. Orso, ai restauri del Priorato quattrocentesco, del campanile medievale e della "Casa del tiglio".

\section{Chiesa di S. Lorenzo}

Lo scavo archeologico, suddiviso in 6 lotti, è stato iniziato nel 1972 e ultimato nel $1986 \mathrm{e}$ ha permesso di riportare alla luce le fasi costruttive della chiesa che brevemente si possono così riassumere ${ }^{7}$ :

- Sistemazione dell'area cimiteriale romana;

- Costruzione, nel secondo quarto del v secolo, della chiesa cruciforme;

- Ricostruzione, a seguito della distruzione del precedente edificio verso la fine dell'viII secolo, causata da un forte incendio, di una piccola chiesetta altomedievale;

- Ricostruzione parziale e ampliamento dell'edificio altomedievale, all'inizio dell'xi secolo;

- Ricostruzione totale della chiesa nella prima metà del XVII secolo. In questa fase la chiesa ruoterà il suo orientamento e l'abside verrà collocata ad ovest per creare il nuovo assetto urbanistico che ancora oggi caratterizza il sagrato esistente tra le due chiese.

L'area di scavo, oltre ad interessare completamente la chiesa attuale, ha contemplato anche le aree e gli edifici esterni che l'attorniavano, in alcuni casi di proprietà privata. La situazione patrimoniale ciha obbligati a soluzioni particolari nei lavori di copertura e sistemazione, al fine di restituire le aree private alla loro originaria funzione e nel contempo assicurare la fruizione al pubblico del sito. Per queste ragioni si sono dovute realizzare due "vele" di copertura dei bracci nord e ovest (fig. 5) in modo tale da garantire l'uso ortivo delle aree annesse ai canonicati interessati. Per permettere ai visitatori la percezione della pianta cruciforme paleocristiana, si è invece proceduto alla demolizione, in corrispondenza dei quattro bracci, delle murature di fondazione della chiesa attuale, in maniera tale da creare in sottosuolo, ma soprattutto visivamente, un vano di fatto cruciforme. 


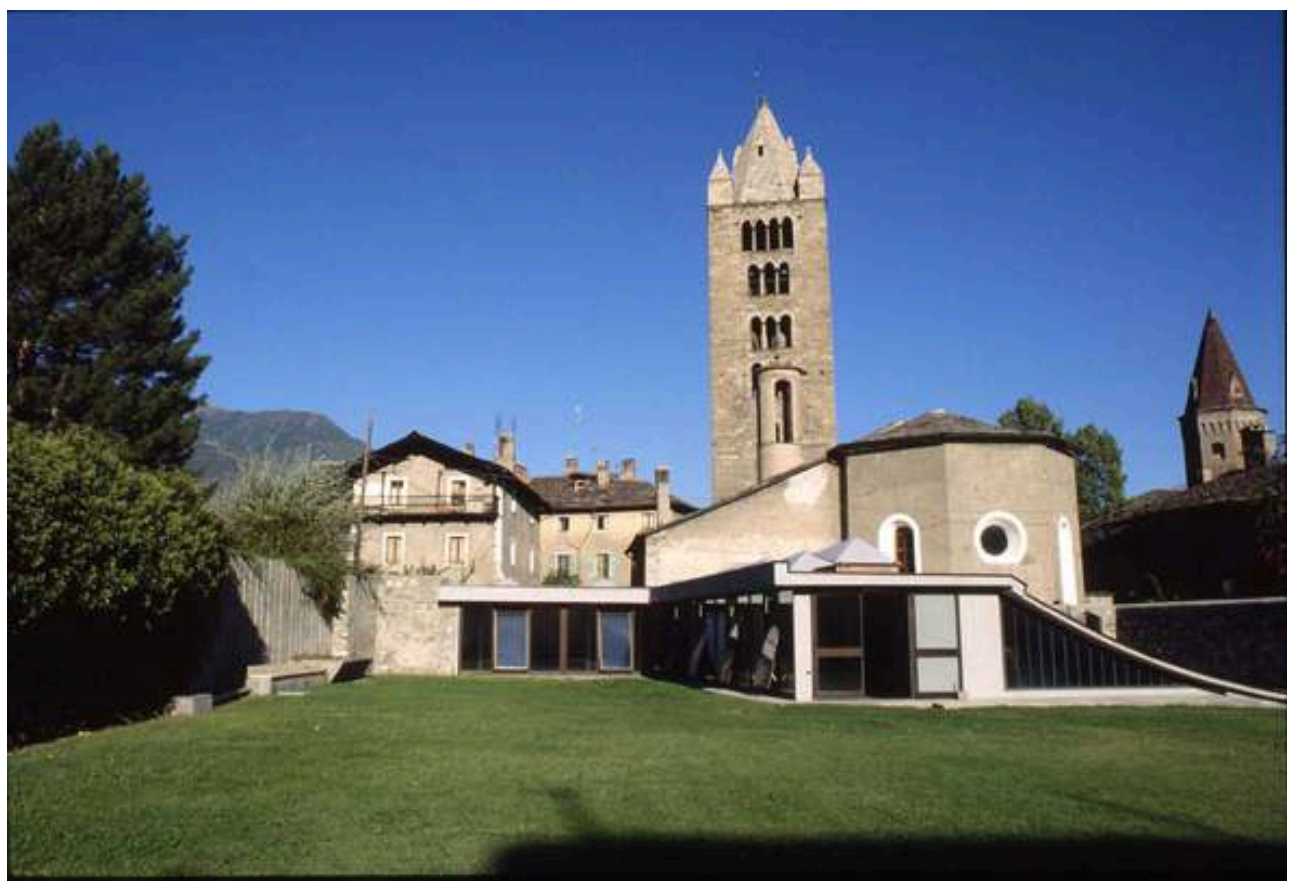

Fig. 5 - Sistemazione scavo S. Lorenzo: veduta esterna.

18 Al fine di migliorare la comprensione delle varie fasi costruttive si sono sacrificati alcuni piccoli tratti delle murature di facciata delle chiese altomedievali e medievali.

19 La grande lacuna, provocata da una profonda fossa di distruzione, situata al centro del presbiterio antico, è stata risarcita con alcune ricostruzioni realizzate in cartongesso pressato colorato, in maniera tale da rendere leggibili, integrandole, le strutture liturgiche e le relative tombe senza per altro intaccare i fragili resti antichi. Queste nuove strutture risultano completamente reversibili e facilmente asportabili. Particolare attenzione è stata anche posta all'accesso dello scavo che tende a riproporre l'antico percorso processionale situato a sud e a ovest della chiesa attuale. La visita prevede una visione dall'alto del braccio occidentale (fig. 6), la discesa nel sottosuolo tramite una scala ubicata a nord dell'abside ovest e l'accesso a una pedana rialzata quasi al centro della chiesa, da cui è possibile osservare le quattro absidi, il presbiterio $\mathrm{e}$ la solea.Uno schermo trasmette invece le immagini delle aree non accessibili ${ }^{8}$. Il ritorno al piano superiore è realizzato lungo il lato occidentale dell'abside nord. 


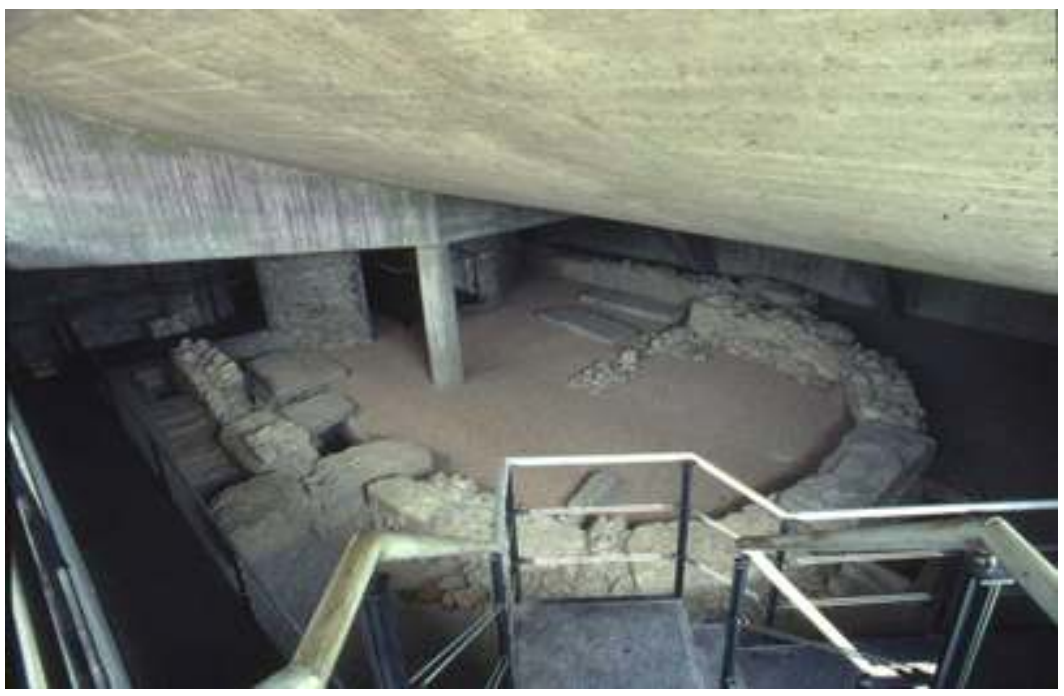

Fig. 6 - Sistemazione scavo S. Lorenzo: vela ovest.

Il sito non è dotato di apparecchiature per il trattamento dell'aria poiché si è verificato che $\mathrm{i}$ valori temperatura-umidità relativa sono compatibili con le esigenze di conservazione.

\section{Chiesa di S. Orso}

Lo scavo archeologico della chiesa di S. Orso, eseguito durante l'arco di nove campagne di scavo, è iniziato nel 1975 ed è stato ultimato nel 2002. Attualmente è però in corso una nuova campagna nell'area meridionale del sagrato. Le ricerche proseguiranno anche nei prossimi anni.

Gli scavi hanno permesso di riportare alla luce importanti resti delle fasi costruttive della Collegiata che brevemente si possono riassumere (vedere figg. 2, 3 e 4):

- Costruzione di un mausoleo con tomba in muratura al centro, risalente al IV secolo;

- Costruzione, nel corso della prima metà del v secolo, di una chiesa funeraria paleocristiana con annesso porticato sui lati nord, ovest ed est.

- Ricostruzione, alla fine dell'viII secolo, di una grande chiesa altomedievale a tre navate concluse da altrettante absidi. Le dimensioni raggiunte dall'edificio in questo periododimostrano l'importanza assunta dal complesso in seguito all'introduzione del culto di Sant'Orso.

- Ricostruzione, tra la fine del x e l'inizio dell'xi secolo, ad opera del vescovo Anselmo, di una chiesa a tre navate e della cripta;

- Costruzione del chiostro e del mosaico del coro (fig. 7), verso la metà del XII secolo;

- Costruzione, nel corso del XIII secolo, del grande campanile isolato;

- Costruzione, verso la fine del xv secolo, del Priorato. 


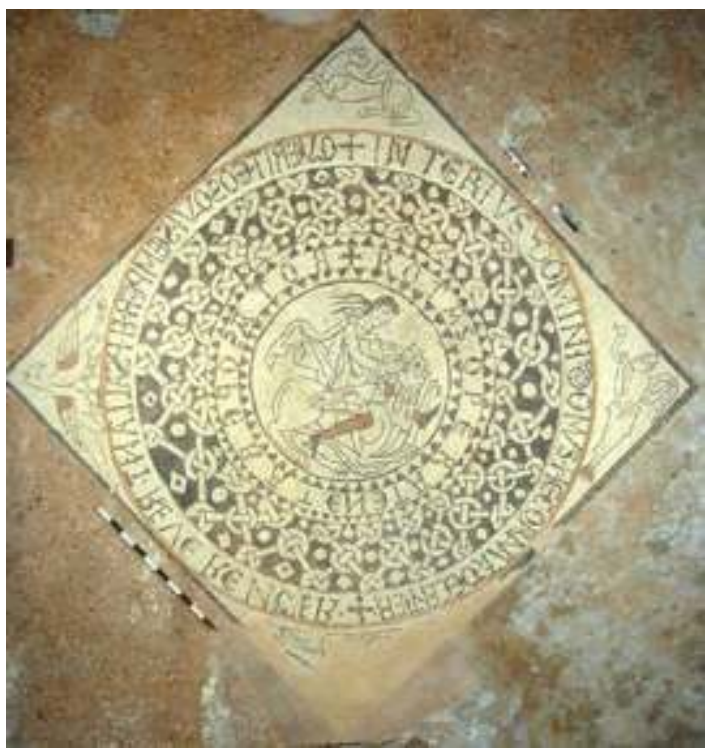

Fig. 7 - Chiesa di S. Orso: mosaico del coro del x॥l secolo.

La chiesa, in epoca paleocristiana, faceva parte di un unico complesso cultuale che comprendeva anche la chiesa di S. Lorenzo ${ }^{9}$.

La differenza di quota, molto contenuta, tra il pavimento attuale e i resti archeologici, non ha permesso una sistemazione a visita del sito, anche in considerazione dell'impossibilità di rialzare il suolo d'uso della chiesa attuale fortemente caratterizzata in età barocca. Per queste ragioni si è proceduto al rifacimento di un pavimento ligneo sostenuto da travi poggianti su piccoli supporti verticali. La soluzione adottata permette però, anche se in maniera poco agevole, di ispezionare il sottosuolo per verifiche e controlli. E' comunque stato possibile rendere fruibile al pubblico l'importante mosaico ${ }^{10}$ della metà del xII secolo (fig. 8), ritrovato nel coro durante la campagna di scavo del 1999 e reso visibile sotto una grande lastra di vetro situata davanti all'altare. E' in corso invece il restauro della parete occidentale della cripta per valorizzare il ritrovamento delle tre aperture che permettevano di vedere le tombe del vano funerario antistante la cripta stessa.

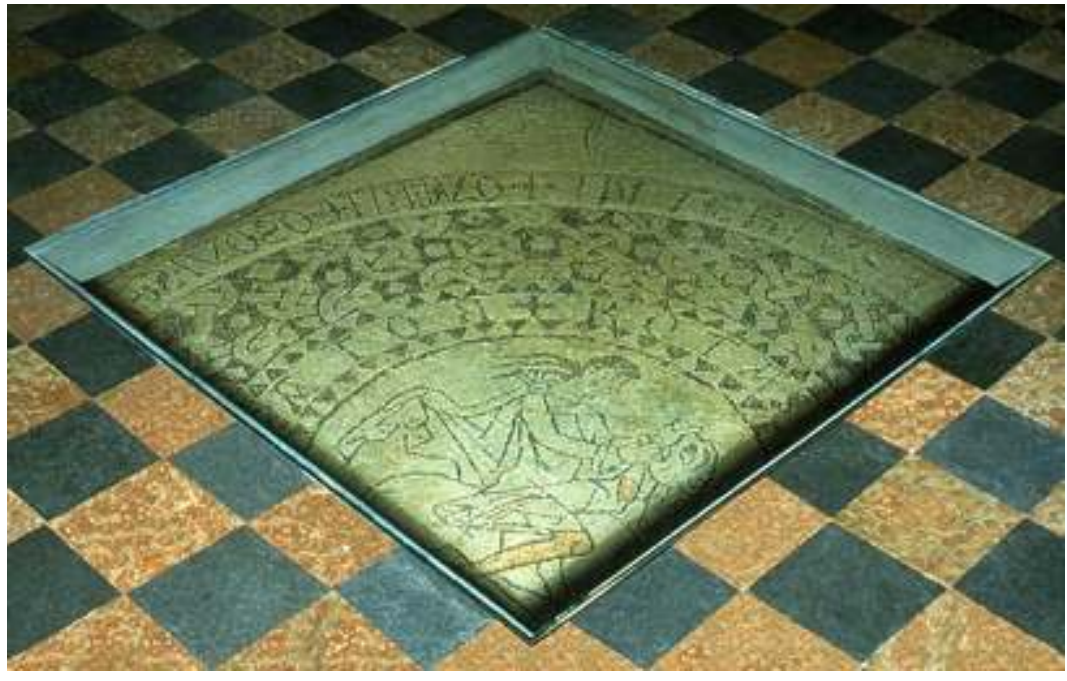

Fig. 8 - Chiesa di S. Orso: sistemazione del mosaico del coro. 
Nel sottotetto della navata centrale, già dal 1968, è possibile ammirare i preziosi affreschi della prima metà dell'xi secolo ${ }^{11}$. La visita è resa possibile tramite una passerella (fig. 9) sostenuta da strutture metalliche e accessibile da una scala a chiocciola costruita ex novo, a est della sacrestia settentrionale.

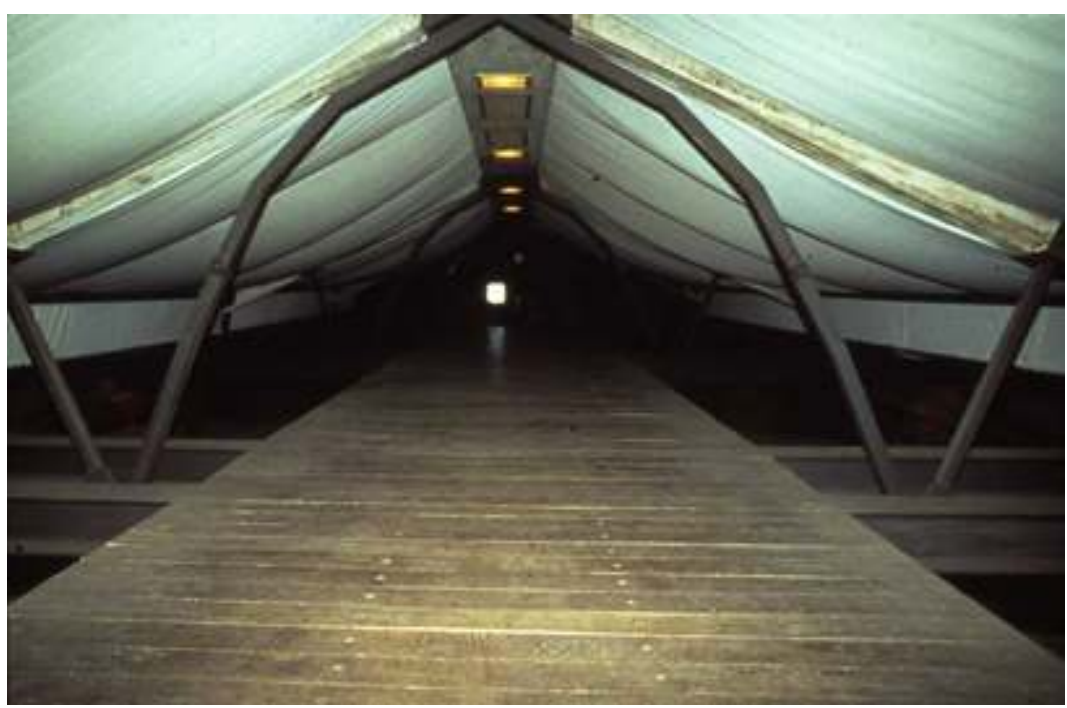

Fig. 9 - Chiesa di S. Orso: passerella sottotetto.

\section{Complesso episcopale}

Lo scavo della Cattedrale, iniziato nel 1976, è tuttora in corso nelle sue aree adiacenti. Le ricerche hanno permesso di ritrovare una sequenza archeologica che copre un arco cronologico compreso tra la protostoria e i giorni nostri ${ }^{12}$. Le fasi costruttive del sito si possono così riassumere (figg. 10 e 11):

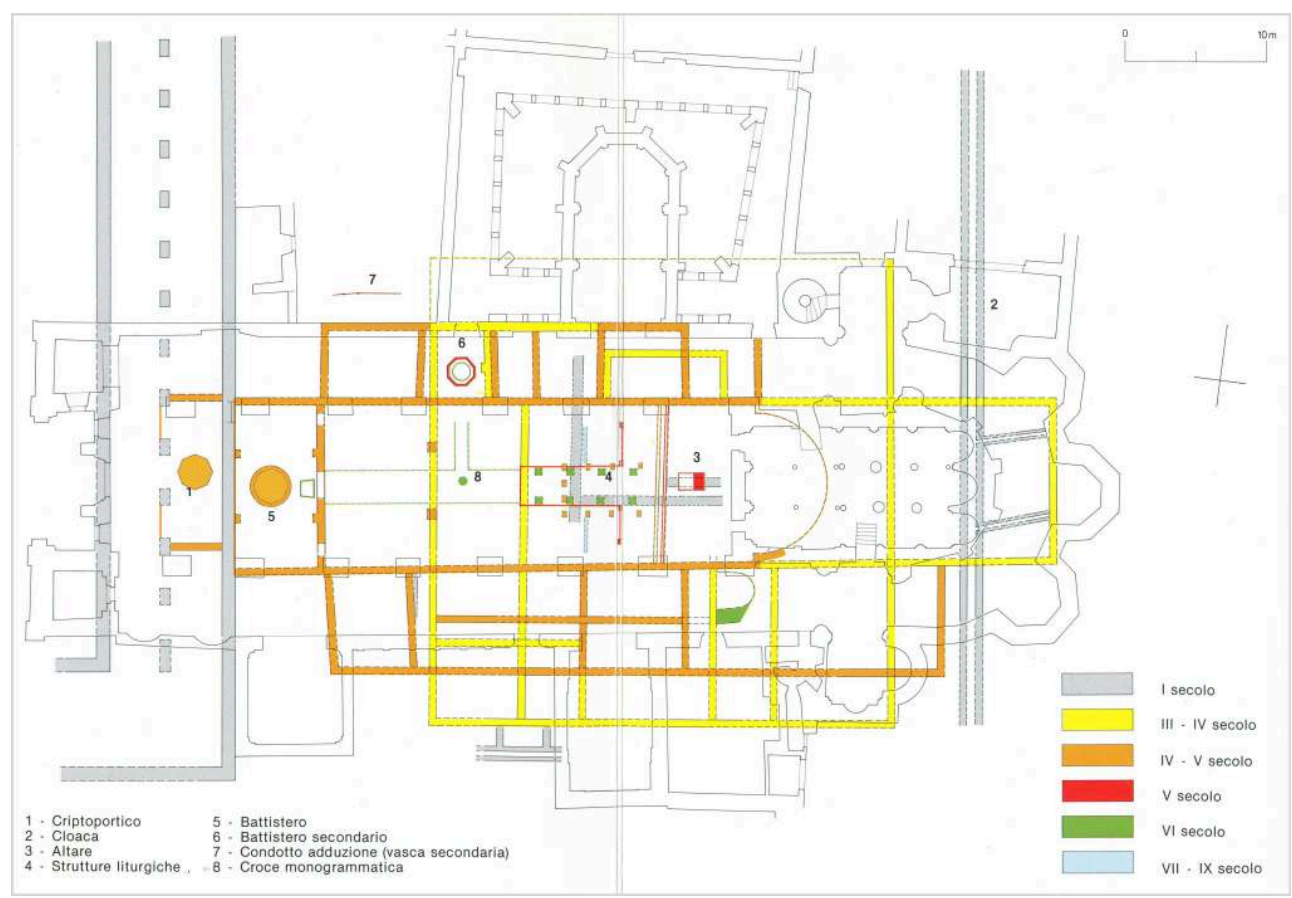

Fig. 10 - Cattedrale: planimetria scavi I-Ix secolo. 


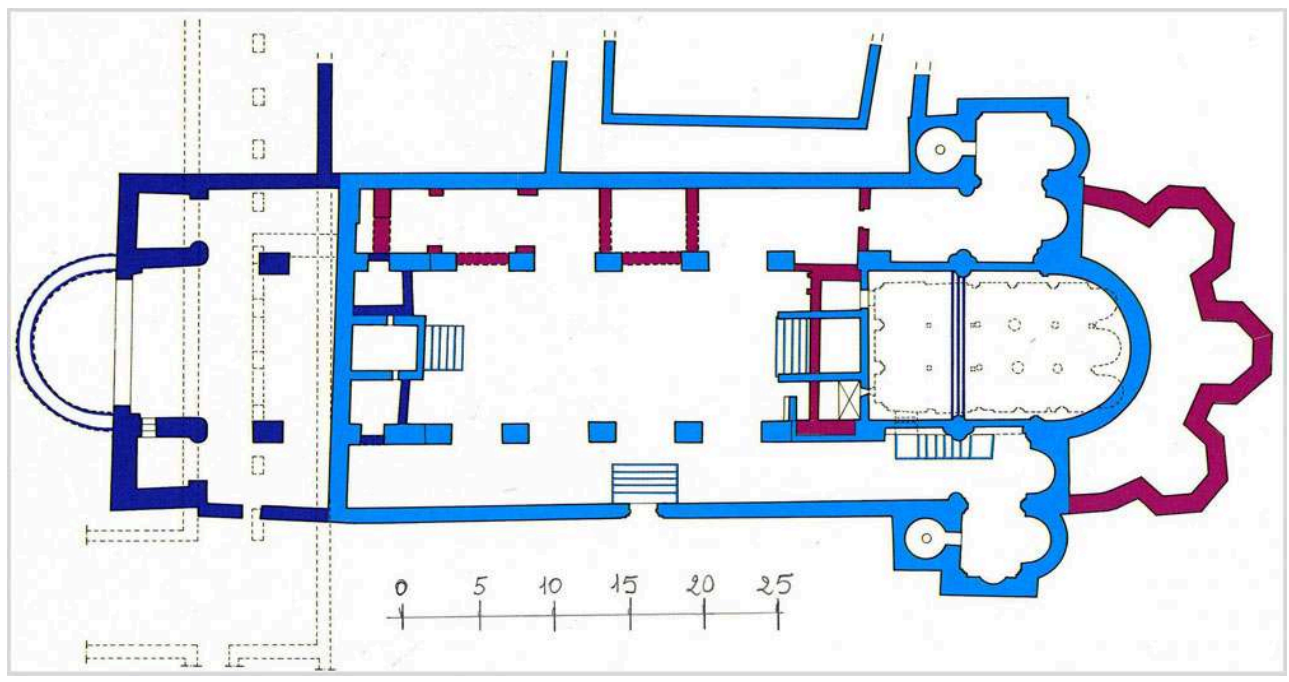

Fig. 11 - Cattedrale: planimetria scavi xI-xIII secolo.

- Ritrovamento di alcuni materiali protostorici venuti alla luce sotto il deambulatorio;

- Costruzione, verso la fine del I secolo a. C., del criptoportico forense;

- Costruzione, tra la fine del I secolo a.C. e l'inizio del I secolo d. C., di una domus situata a est del criptoportico forense:

- Ricostruzione, tra la fine del III e l'inizio del IV secolo, della domus precedente,

- Sistemazione di alcuni ambienti della domus per realizzare una domus ecclesiae e costruzione di una vasca battesimale all'interno dell'ala orientale del criptoportico;

- Costruzione, alla fine del IV secolo, di una cattedrale paleocristiana ad aula unica absidata e dei vani annessi tra cui due battisteri (figg. 12 e 13);

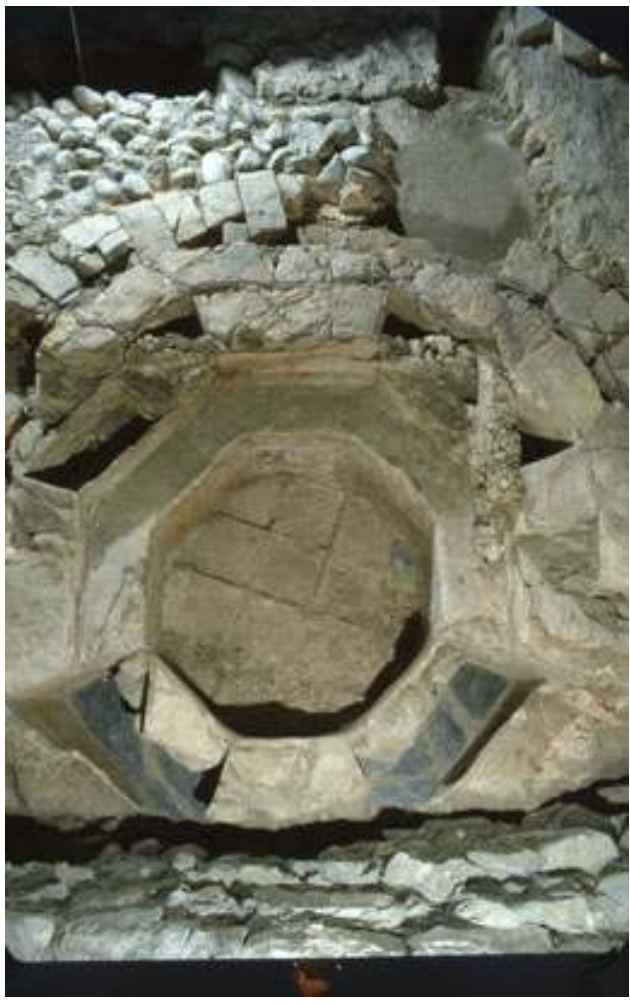

Fig. 12 - Cattedrale: battistero principale. 


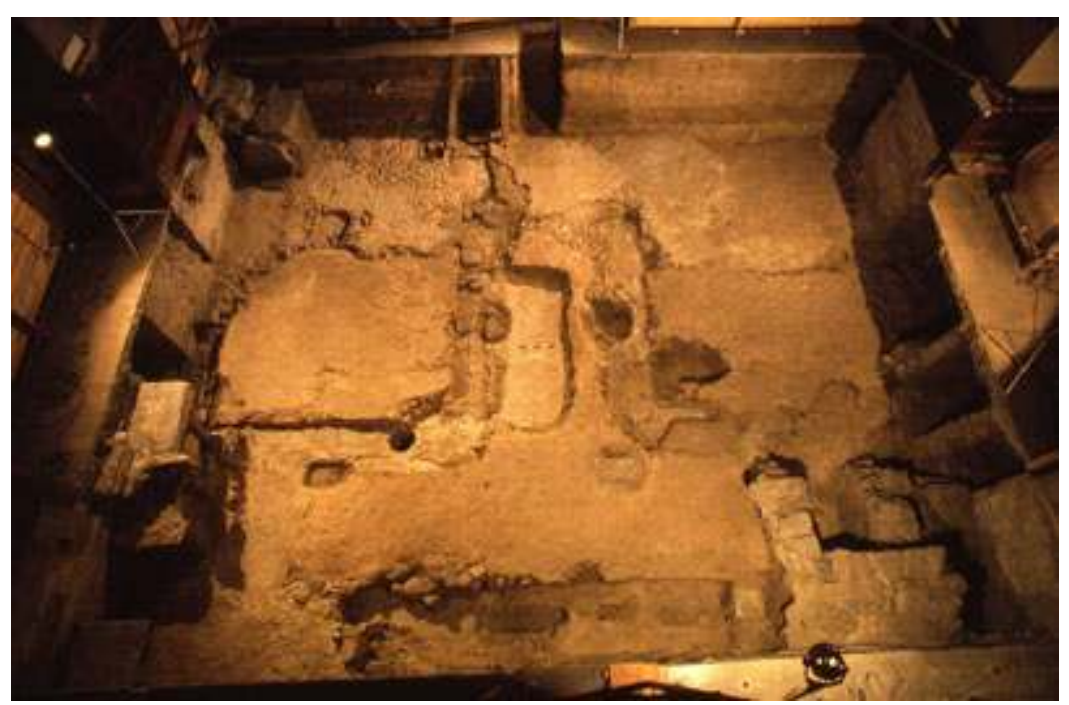

Fig. 13 - Cattedrale: solea.

- Rifacimenti parziali e modificazioni delle vasche battesimali e della solea nel corso del v e vi secolo;

- Ricostruzione, durante il Ix secolo, di parte del presbiterio;

- Ricostruzione totale della chiesa, per opera del vescovo Anselmo, all'inizio dell'xi secolo;

- Costruzione, nella seconda metà dell'xi secolo, del massiccio occidentale;

- Costruzione, alla fine del xIII secolo, del Jubé e del deambulatorio;

- Modificazioni tra il XIII e il xx secolo.

Lo scavo della cattedrale rientra in un vasto programma di ricerche e valorizzazione che coinvolge un'area di circa $45.000 \mathrm{mq}$ situata al centro dell'antica Augusta Praetoria e comprende le due insulae a nord-est del Decumano e Cardo massimi con il foro, il criptoportico forense con i templi, la grande esedra settentrionale, la Porta Principalis Sinistra, la Cattedrale, il chiostro quattrocentesco e alcuni canonicati. A lavori ultimati la città potrà disporre di un sito visitabile di notevole estensione e di grande interesse scientifico e archeologico, che illustrerà le vicende costruttive dell'area dal periodo augusteo ai giorni nostri con le relative modificazioni urbanistiche.

A tutt'oggi sono visitabili la cattedrale con il Museo del tesoro, gli scavi, il chiostro, il sottotetto con gli affreschi dell'xi secolo, il criptoportico, il foro e l'area sottostante il Museo regionale. A breve sarà redatto e realizzato un progetto complessivo di visita che ha come obiettivo l'aggregazione di tutti gli scavi fin'ora realizzati e la loro valorizzazione contestualmente ai monumenti ancora esistenti in elevato. 


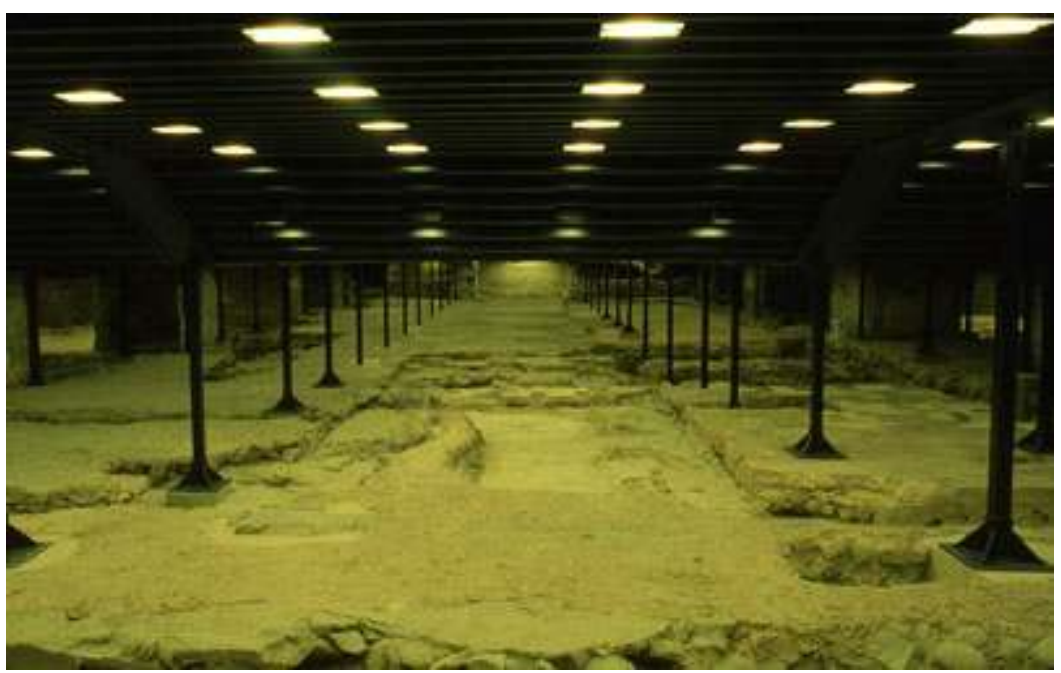

Fig. 14 - Cattedrale. Sistemazione scavi navata principale.

Il sottosuolo della cattedrale è stato sistemato già a partire dal 1986 (fig. 14). Esso è accessibile dal criptoportico, dalla cripta, dalla cappella dei signori di Cly situata a sud dell'ingresso principale e da una scaletta a sinistra del coro attuale. Il percorso di visita si snoda circolarmente, sotto il pavimento della chiesa, lungo una passerella che percorre le navate laterali (fig. 15), il criptoportico (fig. 16) a ovest e l'area antistante il coro. Il percorso sfrutta le canalizzazioni di un impianto di riscaldamento realizzato, all'inizio del xx secolo, distruggendo parte dei suoli d'uso dell'antica cattedrale. Il cunicolo era stato individuato, ispezionato e oggetto di alcuni piccoli saggi di scavo, prima dell'inizio delle ricerche in estensione. Particolare attenzione è stata dedicata a far si che tutti gli scavi venissero eseguiti mantenendo le attività liturgiche all'interno della chiesa. Per questa ragione la soletta portante il pavimento, è stata realizzata con strutture prefabbricate costituite da una lamiera grecata con funzione autoportante sulla quale è stato steso un getto in cemento armato. La soletta è sostenuta da pilastrini a sezione quadrata di piccole dimensioni tali da garantire però una buona visibilità al contesto archeologico. La passerella è realizzata con ringhiere in acciaio inox e pavimento in lamiera sul quale è statocollocato un piccolo strato di resina caricata con sabbia di quarzo in modo da eliminare qualsiasi rumorosità durante il transito delle persone. Il mancorrente delle ringhiere è attrezzato per contenere la distribuzione degli apparecchi audiovisivi ed elettrici. L'intradosso della soletta è stato dipinto in blu scuro al fine di diminuirne l'impatto; negli incavi della lamiera sono stati inseriti gli apparecchi illuminanti e di sicurezza. 


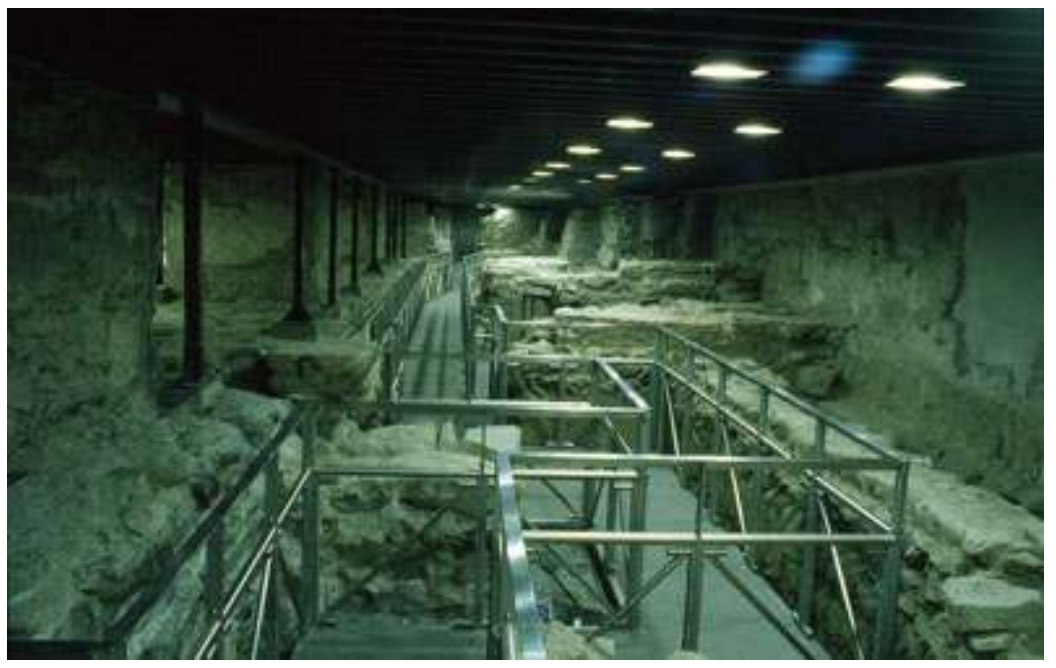

Fig. 15 - Cattedrale: passerella navata nord.

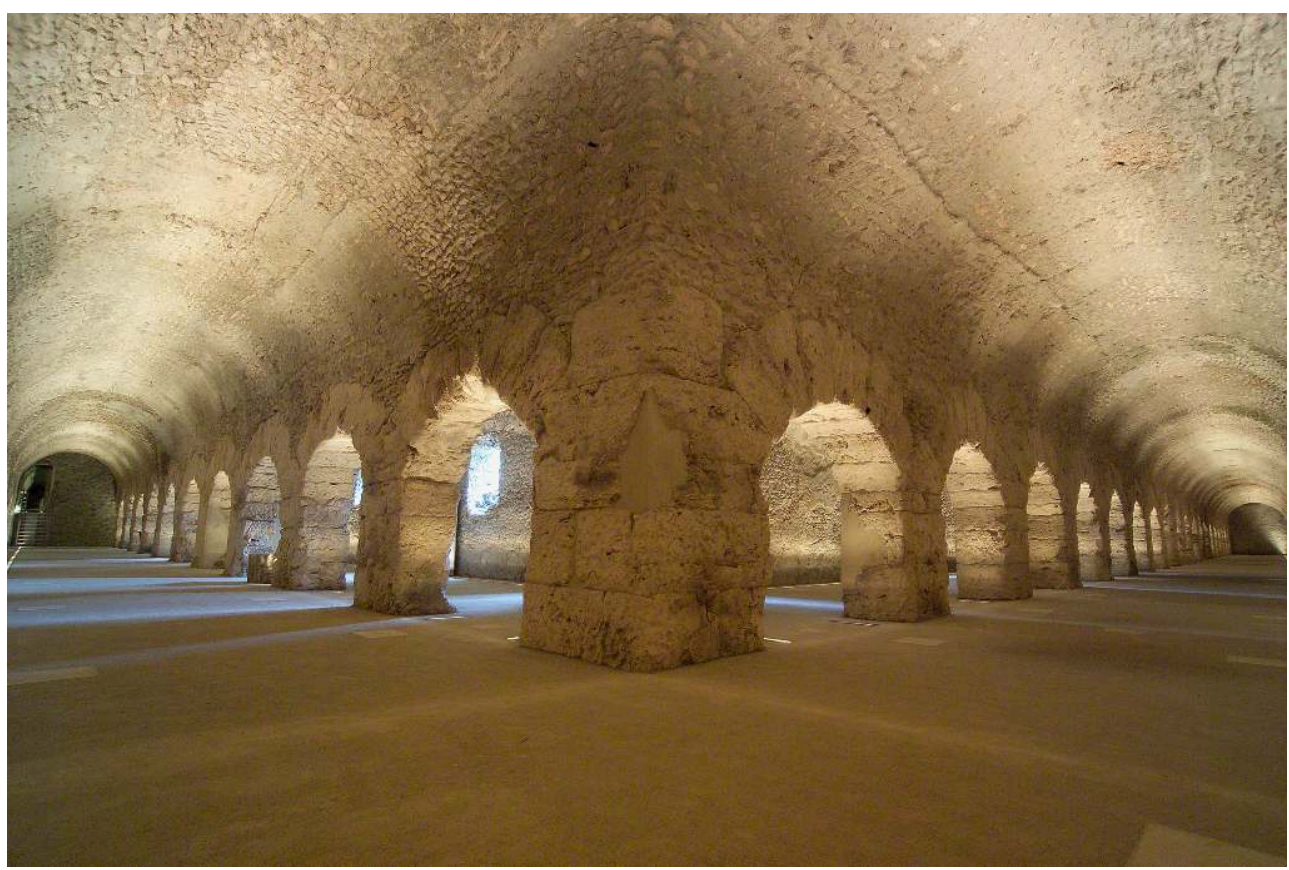

Fig. 16 - Criptoportico forense.

30 Per ragioni legate alla fruizione si è privilegiata la fase paleocristiana evitando di distruggere i suoi due pavimenti a scapito però della conoscenza delle fasi costruttive della domus romana, indagata solamente svuotando le lacune dei pavimenti causate dalle fosse di fusione quattrocentesche di alcune campane.

31 Contestualmente ai lavori archeologici sono stati realizzati, il nuovo Museo del tesoro, ubicato nel deambulatorio del XIII secolo, e la passerella (fig. 17) per la visita degli affreschi (fig. 18) ancora visibili nel sottotetto della navata centrale e accessibili utilizzando la torre scalare del campanile settentrionale. 


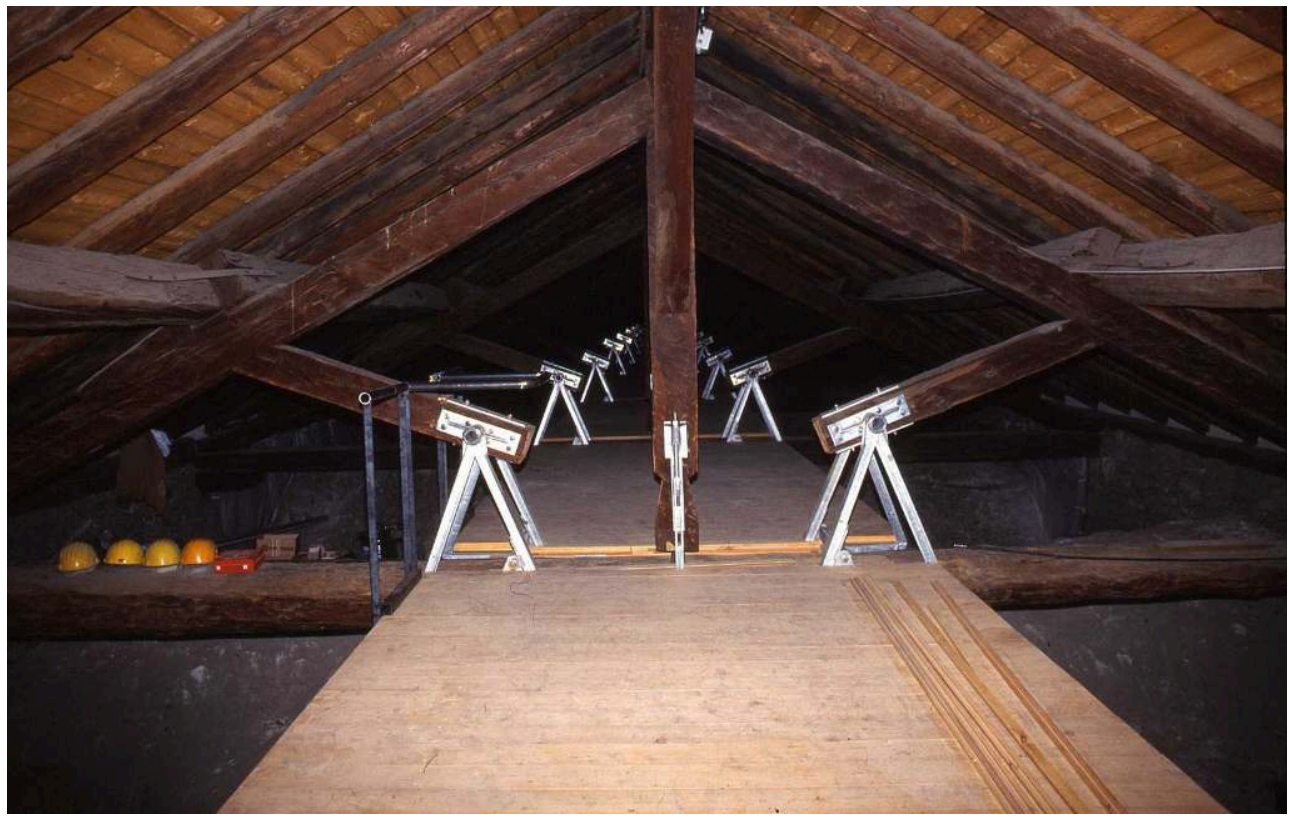

Fig. 17 - Cattedrale: passerella sottotetto navata centrale.

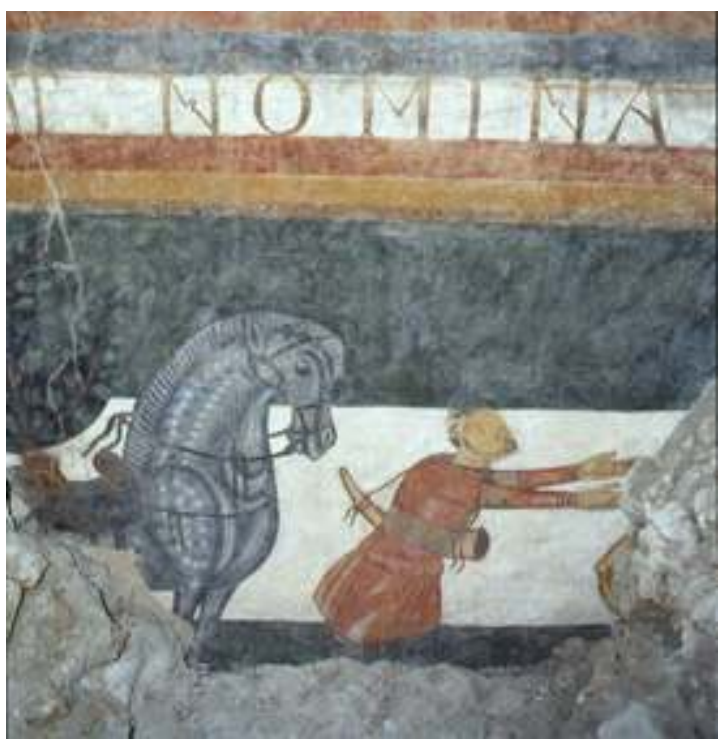

Fig. 18 - Cattedrale: affreschi sottotetto.

La passerella, contrariamente a quanto precedentemente realizzato a S. Orso, ha mantenuto in situ le capriate di sostegno del tetto, asportando unicamente un piccolo tratto dei puntoni per poter sfruttare un'altezza minima superiore a m 1,80. I lavori sono stati eseguiti senza smontare il tetto da poco tempo rifatto.

Come si può notare da quanto descritto, la visita al monumento avviene su tre livelli, sottosuolo con cripta, poi la chiesa, il museo e il chiostro, infine il sottotetto e le cappelle alte.

\section{Conclusione}

34 La conservazione e sistemazione dei resti archeologici dei due complessi aostani, ha comportato ingenti impegni finanziari diluiti lungo ben quattro decenni, con disagi ai 
cittadini e soprattutto ai fedeli. Si ritiene però che la ricaduta, in termini turistici, possa compensare le risorse spese.

La ricerca archeologica e la valorizzazione dei siti legati alla storia del cristianesimo assume, in questo momento storico, grande importanza, in considerazione del lento e continuo abbandono delle pratiche religiose da parte di quasi tutti i cittadini europei, con conseguente perdita delle pratiche religiose e delle loro tracce presenti nelle chiese. Fra non molti decenni, perduta la pratica del battesimo, potremmo trovarci a dover spiegare ai visitatori che cos'è un fonte battesimale e un battistero! A queste considerazioni bisogna anche considerare che con l'accentuarsi dei flussi migratori nuove dottrine religiose si vanno sempre più diffondendo, come ad esempio la religione islamica, implicando radicali cambiamenti culturali.

\section{NOTES}

1. La Regione Autonoma della Valle d'Aosta, in virtù dello Statuto Speciale ottenuto con la legge Costituzionale 26 febbraio 1948, n. 4, ha potestà legislativa integrativa nel campo delle Antichità $e$ Belle Arti ed esercita le funzioni amministrative degli organi dello Stato nella materia di cui sopra. L'Amministrazione Regionale ha organizzato le tre tradizionali competenze delle Soprintendenze dello Stato (beni archeologici, architettonici e storico-artistici) in un'unica Soprintendenza, pur accettando di creare delle specializzazioni interne inevitabili per la qualificazione degli interventi, ma evitando di creare un'autonomia decisionale dei singoli settori e soprattutto cercando di affrontare ogni intervento valendosi di apporti interdisciplinari. In accordo con questo indirizzo il Soprintendente è il dirigente unico del Servizio. Al riguardo vedere: R. PERINETTI, L'esperienza della Soprintendenza regionale della valle d'Aosta, in I Beni Culturali ed Ambientali tra Stato ed Enti Locali, Aosta, 2000, pp. 35-37; F. MONTANARI e R. PERINETTI, L'esperienza della Sovrintendenza regionale della Valle d'Aosta, in Monumenti. Una risorsa per il futuro. ICOMOS Comitato Italiano. Contributi preliminari. Roma, 24-25 maggio 1989,Napoli, 1989, pp. 223-234.

2. In Cattedrale ad esempio si sono conservati i suoli d'uso dell'aula sacrificando però la conoscenza della domus del i secolo d. C.

3. J.-C. GARDIN, Les considérations d'économie en archéologie, in Coût et profits en archéologie, CNRS, Paris, 1980.

4. R. PERINETTI e S. PULGA, I siti archeologici della Valle d'Aosta: problemi ed esperienze, in I siti archeologici. Un problema di musealizzazione all'aperto. Primo seminario di studi. Roma, febbraio 1988. Provincia di Roma. Assessorato alla Pubblica Istruzione. Roma, 1988, pp. 213-217.

5. Il programma di ricerca prevedeva anche lo scavo delle altre chiese funerarie della città (S. Stefano e basilica fuori Porta Decumana) e delle chiese battesimali di alcuni centri rurali della Regione (Villeneuve e Morgex). Al riguardo vedere: R. PERINETTI, Il Battistero Paleocristiano della Chiesa Santa Maria di Villeneuve, in Bulletin d'Etudes Préhistoriques Alpines. XV, Aoste, 1983, pp. 205-213; R. PERINETTI, Gli Edifici Paleocristiani di Villeneuve (Aosta), in Atti del VI Congresso Nazionale di Archeologia Cristiana. Pesaro - Ancona, 19-23 settembre 1983. Ancona, 1985, pp. 809-823. R. PERINETTI, chiesa S. Maria di Villeneuve. Risultati delle prime campagne di scavo, in Bollettino dell'Accademia di Sant'Anselmo. I (Nuova serie). Aosta, 1985, pp. 160-198; Ch. BONNET et R. PERINETTI, en collaboration avec M. CORTELAZzo, Deux novelles églises paléochrétiennes de la Vallée d'Aosta, in Rivista 
di Archeologia Cristiana. Anno LXXX, Città Del Vaticano, 2004 (pubbl. 2005), pp. 159-194; R. PERINETTI, Valle d'Aosta - Le chiese altomedievali, in Alle origini del romanico. Monasteri, edifici religiosi, committenza tra storia e archeologia (Italia settentrionale, secoli IX-X), a cura di R. SALVARANI, G. ANDENNA e G. P. BRogiolo, Brescia, 2005, pp. 149-164.

6. A. P. FRUTAZ, Le fonti per la storia della Valled'Aosta, in Thesaurus Ecclesiarum Italiae I, I, Roma, 1966, pp. 5-8 e 289-292.

7. Ch. BONNET avec la collaboration de R. PERINETTI, Saint-Laurent d'Aoste. Rapport préliminaire des fouilles de 1972, in Duria - Rivista della Soprintendenza Regionale ai Monumenti, Antichità e Belle Arti - Vol. I - 1974, Torino 1975; Ch. BONNET, L'église cruciforme de Saint-Laurent d'Aoste. Etude archéologique (les fouilles de 1972 à 1979), in Quaderni della Soprintendenza per i beni culturali della Valle d'Aosta 1. Nuova serie. La chiesa di San Lorenzo in Aosta. Scavi archeologici, Roma, 1981, pp.11-46; R. PERINETTI, La chiesa di San Lorenzo. Appunti per una tipologia delle tombe, in Quaderni della Soprintendenza..., pp. 47-92; R. PERINETTI, Valle d'Aosta - Le absidi dei primi edifici cristiani, in Bulletin d'Études Préhistoriques Alpines. XVIII, Aoste, 1986, pp. 99-114; Ch. BONNET et R. PERINETTI, Aoste aux premiers temps chrétiens, Quart (Aoste), 1986. R. PERINETTI, Le tombre privilegiate della chiesa di S. Lorenzo ad Aosta in L'inhumation privilégiée du $\mathrm{IV}^{\mathrm{e}}$ au VIII ${ }^{\mathrm{e}}$ siècle en Occident. Actes du Colloque tenu à Créteil les 16-18 mars 1984, éditées par Y. DuvAL et J.-Ch. PICARD, Paris, 1986, pp.143-156; Ch. BONNET en collaboration avec M. PERINETTI, Les premiers édifices chrétiens d'Augusta Praetoria (Aoste, Italie), in Académies des Inscriptions \& Belles-Lettres. Comptes Rendus des séances de l'année 1986. Juillet-octobre, Paris, 1986, pp. 477-496 ; R. PERINETTI, Augusta Praetoria - Le necropoli, le sepolture e i riti funerari cristiani, in Aosta. Progetto per una storia della città. A cura di Marco Cuaz, Quart (Aosta), 1987, pp.121-163; R. PERINETTI, Valle d'Aosta, in Actes du XI Congrès International d'Archéologie Chrétienne. Lyon, Vienne, Grenoble, Genève, Aoste, 21-28 septembre 1986. Volume 3. Appendici, Città del Vaticano, 1989, pp. 2245-2253; R. PERINETTI, Augusta Praetoria: Le necropoli cristiane, in Actes du XI ${ }^{e}$ Congrès International d'Archéologie Chrétienne, Lyon, Vienne, Grenoble, Genève, Aoste, 21-28 septembre 986. Volume 2, Città del Vaticano, 1989, pp.1215-1226; J.-F. ReYNAUD, R. COLARDELLE, M. JANNETVALLAT, R. PERINETTI et B. PRIVATI, Les édifices funéraires et les nécropoles dans les Alpes et la Vallée du Rhône. Origines et premiers développements, in Actes du XI ${ }^{\mathrm{e}}$ Congrés International..., pp. 1475-1514; R. PERINETTI, Le sepolture nella chiesa di San Lorenzo ad Aosta. Cronologia e tipologia delle tombe, in Mediterraneo tardoantico e medievale. Scavi e ricerche. 8. Le sepolture in Sardegna dal IV al VII secolo. IV Convegno sull'archeologia tardoromana e medievale (Cuglieri, 27-28 giugno 1987), Oristano, 1990, pp.335-382; R. PERINETTI, Le sepolture cristiane in Valle d'Aosta (sec. V-XVIII): Cronologia relativa e assoluta, in Bulletin d'Études Préhistoriques et Archéologiques Alpines. Actes du V $\mathrm{V}^{\mathrm{e}}$ Colloque sur les Alpes dans l'Antiquité. Pila, Vallée d'Aoste, 11-13 septembre 1987, Aoste 1990, Quart (Vallée d'Aoste), pp. 219-279 ; R. PERINETTI, Valle d'Aosta. Riti e Pratiche Funerarie Cristiani, in Bulletin d'Etudes Préhistoriques et Archéologiques Alpines. Actes du VI ${ }^{\mathrm{e}}$ Colloque International sur les Alpes dans l'Antiquité. Annecy, Haute-Savoie. 23-24 septembre 1989. II Aoste 1991, Quart (Vallée d'Aoste), 1991, pp. 89-114; A. M. CAVAllaro e R. PERINETTI, Tre nuove iscrizioni da Aosta, in Miscellanea epigrafica in onore di Lidio Gasperini, Tivoli (Roma), 2000, pp. 222-230;

8. F. Montanari e G. Vallacqua, La sistemazione di visita dello scavo di S. Lorenzo in Aosta. Problemi di architettura e di struttura. In Quaderni della Soprintendenza ..., pp. 123-135.

9. R. PERINETTI, Aosta. La chiesa dei SS. Pietro e Orso, in Acta Congressus Internationalis XIV Archaeoligiae Christianae. Vindobonae 19, - 26. 9. 1999. Pars I, Città Del Vaticano, 2006, pp. 589-608; Ch. BONNET e R. PERINETTI, La Collegiata di Sant'Orso dalle origini al XIII secolo, in Sant'Orso di Aosta. Il complesso monumentale. Volume I. Saggi. A cura di B. ORLANDONI e E. ROSSETTI BREZZI, Aosta, 2001, pp. 9-34; 
10. R. PERINETTI, I mosaici medievali di Aosta, in AISCOM. Atti del VI Colloquio dell'Associazione Italiana per lo studio e la conservazione del mosaico. Venezia, 20-23 gennaio 1999, Ravenna, 2000, pp. 161-174.

11. AA. VV., Medioevo Aostano. La pittura intorno all'anno 1000 in Cattedrale e in Sant'Orso. Volume I. Atti del Convegno Internazionale. Aosta 15-16 maggio 1992. Volume II. Atlante fotografico, Torino 2000, raccolti da S. Barberi.

12. Ch. Bonnet e R. PERINETTI, Remarques sur la crypte de la Cathédrale d'Aoste, in Quaderno n. 1.Conservazione dei beni culturali. Sopritendenza ai beni culturali della Valle d'Aosta, Aoste, 1977; Ch. BONNET et R. PERINETTI, Aoste aux..., Quart (Aoste), 1986 ; Ch. BONNET en collaboration avec M. PERINETTI, Les premiers édifices chrétiens..., pp.477-496; R. PERINETTI, La Cattedrale anselmiana, in Seminari di Archeologia Cristiana (Archeologia e Cultura della Tarda Antichità e Alto Medioevo). Resoconto delle sedute dell'A.A. 1992-1993. Rivista di Archeologia Cristiana N. 1-2- anno LXIX- 1993, Roma, 1993; Ch. Bonnet, R. PERINETTI, I battisteri della cattedrale di Aosta, in Albenga Città episcopale. Tempi e dinamiche della cristianizzazione tra Liguria di Ponente e Provenza. Atti del Convegno internazionale - Tavola rotonda. Albenga 21-23 settembre 2006, Genova - Albenga, 2007, pp. 821-837.

\section{INDEX}

Mots-clés : groupe épiscopal

Index géographique : Italie/Aoste 